\title{
Introduction
}

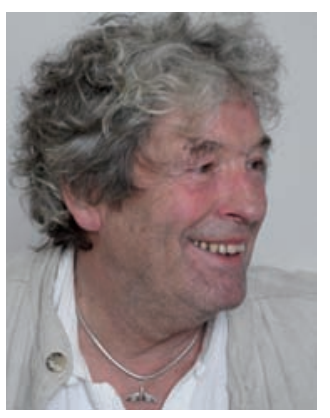

\section{_Dominique Delfieu}

Cabinet médical, 85 rue Lepic, 75018 Paris

docdom@aol.com
$P$ our ce dernier dossier thématique d'Oncomagazine, il nous est apparu opportun de traiter de ces situations où la maladie cancéreuse vient se surajouter à un handicap, une fragilité, une pathologie qui vont potentialiser les difficultés que rencontreront le patient et le médecin dans le cours du traitement. Ainsi, il n'existe que très peu d'études validées sur les conduites à tenir lorsque le patient présente outre son cancer, une maladie d'Alzheimer, une pathologie psychiatrique, une tentative de suicide, un handicap qui, par exemple, l'empêchera de se déplacer et de suivre son traitement de façon optimum. Et que dire des enfants qui n'étant pas majeurs, n'ont pas à donner leur accord, sont parfois "sur-traités » et souffrent en dépit de l'absence de bénéfice escompté. On sait déjà que tous les cas de cancer sont difficiles et que chacun est spécifique, aussi l'équipe pluridisciplinaire qui va le soigner doit être bien informée de l'environnement psychosocial dans lequel évolue la personne malade du cancer.

La question de «l'acharnement thérapeutique » fait souvent irruption dans ces situations, car si l'on s'accorde à penser que l'exis- tence synchrone d'une seconde pathologie grave ne doit pas engendrer de perte de chance dans le traitement du cancer, il est bien évident que la mise en œuvre de celui-ci et son observance vont parfois produire de telles difficultés que certains s'interrogeront sur son but et son utilité. C'est dans ce contexte aussi que la place du médecin généraliste doit prendre toute sa valeur au sein de l'équipe traitante, car il connaît les comorbidités, l'entourage, et la situation propre du patient, ce qui lui permettra de tenir une place de conseil auprès des autres soignants, surtout si les interfaces avec les milieux hospitaliers sont fluides et si chacun accepte de remplir son rôle avec ses possibilités, ses contraintes et à l'intérieur de ses limites. En fait, l'organisation de la cancérologie autour de ces équipes multidisciplinaires est assez récente et les champs d'intervention des différents professionnels de santé sont encore à mieux préciser si l'on veut améliorer la continuité et la coordination des soins. De même, la notion de " secret professionnel partagé » doit être scrupuleusement respectée pour éviter certaines dérives d'indiscrétion auxquelles sont exposés nos patients. 